\title{
Influence of Isotopologue Dipole Moments on Precision Dielectric-Constant Measurements
}

\author{
Allan H. Harvey \\ National Institute of Standards and Technology, \\ Boulder, CO 80305, USA \\ allan.harvey@nist.gov
}

Measurements of the relative permittivity (static dielectric constant) of fluids such as methane have been interpreted with the assumption of zero dipole moment. This assumption is not strictly true, due to the presence of isotopologues with small, nonzero dipole moments. We investigate the significance of this effect by analyzing the effect of the dipole of $\mathrm{CH}_{3} \mathrm{D}$ on the static dielectric constant of methane. It is found that the isotopologue effect is more than two orders of magnitude smaller than the uncertainty of the best existing measurements. Similar estimates for other compounds such as $\mathrm{H}_{2}$ and $\mathrm{CO}_{2}$ produce even smaller effects. Therefore, the interpretation of these measurements with a dipole moment of zero remains valid.

Key words: dielectric constant; isotopes; methane; relative permittivity.

Accepted: October 30, 2019

Published: November 4, 2019

https://doi.org/10.6028/jres.124.031

\section{Introduction}

Measurements of the static dielectric constant (relative permittivity) of fluids have a variety of uses in metrology. Many of these applications exclusively employ noble gases, such as dielectric-constant gas thermometry [1]. However, measurements of molecular species ( $>1$ atom) are also of interest, for example in studies of humidity [2] and natural gas [3].

In the early 2000s, Moldover and coworkers [4, 5, 6] published highly accurate capacitance-based measurements of the dielectric constant of several common gases, including the major components of natural gas, at pressures up to $7 \mathrm{MPa}$. For nonpolar fluids, the static dielectric constant $\varepsilon$ is described by the Clausius-Mossotti equation:

$$
\frac{\varepsilon-1}{\varepsilon+2}=\rho A_{\varepsilon}
$$

where $\rho$ is the molar density and $A_{\varepsilon}$ is proportional to the mean zero-frequency polarizability of an isolated molecule. Additional terms of higher order in density can be added to the right-hand side of Eq. (1) [1, 7], but extrapolating dielectric-constant data to zero density can yield accurate values of $A_{\varepsilon}$ (and thence the molecular polarizability). Alternatively, if the polarizability is known, accurate dielectric measurements can determine the molar density. In the studies of Moldover and coworkers [4, 5, 6], $A_{\varepsilon}$ for nonpolar gases such as $\mathrm{N}_{2}, \mathrm{CH}_{4}, \mathrm{C}_{2} \mathrm{H}_{6}, \mathrm{CO}_{2}$, and $\mathrm{H}_{2}$ was typically determined with a relative standard uncertainty on the order of $5 \times 10^{-5}$. 
However, the assumption that the dipole moment is zero is not strictly true for "nonpolar" molecular fluids. The moment is identically zero by symmetry only if the fluid is isotopically pure. Isotopic substitution, for example the substitution of an ordinary hydrogen atom $\left({ }^{1} \mathrm{H}\right)$ by an atom of deuterium $\left({ }^{2} \mathrm{H}\right.$, usually written D), can create a species with a nonzero dipole moment. Two effects contribute to this. First, the unequal nuclear masses create asymmetry in the electron distribution when higher-order effects beyond the Born-Oppenheimer approximation are considered; this happens for example in the HD molecule. Second, and more significant when it occurs, for molecules with more than one intramolecular bond the different reduced masses of the bonds (for example, a C-D bond versus a C-H bond in $\mathrm{CH}_{3} \mathrm{D}$ ) cause asymmetry in the vibrationally averaged molecular geometry and lead to a dipole moment.

Because of the high precision of recent dielectric-constant measurements, it is plausible that the small dipole moments of naturally occurring isotopologues could influence the results. In this brief note, we analyze this question, focusing on the case of $\mathrm{CH}_{3} \mathrm{D}$ in methane.

\section{Analysis}

For describing the low-density behavior of the static dielectric constant of polar fluids, an additional term must be added to Eq. (1); this is sometimes called the Debye-Langevin equation [8]:

$$
\frac{\varepsilon-1}{\varepsilon+2}=\rho\left(A_{\varepsilon}+\frac{A_{p}}{T}\right),
$$

where $T$ is the absolute temperature. The quantity $A_{p}$ is related to the average of the square of the molecular dipole moment $p$ by

$$
A_{p}=\frac{N_{\mathrm{A}}\left\langle p^{2}\right\rangle}{9 \varepsilon_{0} k},
$$

where $N_{\mathrm{A}}$ is the Avogadro constant, $\varepsilon_{0}$ is the electric constant (permittivity of free space), and $k$ is the Boltzmann constant. In Eqs. (2) and (3) and the remainder of this paper, we have used the symbols $p$ and $A_{p}$ for dipole-related quantities instead of the $\mu$ and $A_{\mu}$ used in previous work [3, 5]; this is to avoid confusion with the use of $\mu$ for the magnetic permeability in refractive-index gas metrology [7]. For a mixture of $n$ components (whether different chemical compounds or isotopologues), the average appearing in $A_{p}$ is given by

$$
\left\langle p^{2}\right\rangle=\sum_{i=1}^{n} x_{i} p_{i}^{2}
$$

where $x_{i}$ is the mole fraction and $p_{i}$ the dipole moment of species $i$. To evaluate the significance of isotopologue dipole moments, the magnitude of the $A_{p}$ term in Eq. (2) must be compared to the uncertainty with which $A_{\varepsilon}$ is known. This calculation requires the mole fractions $x_{i}$ and dipole moments $p_{i}$ of the species with nonzero dipole moments.

We now focus on methane, $\mathrm{CH}_{4}$, which preliminary examination of available information suggested would have the largest isotope effect because $\mathrm{H} / \mathrm{D}$ substitutions tend to produce the most asymmetry and $\mathrm{CH}_{4}$ has four opportunities for substitution.

While there is significant natural variability in the isotopic composition of methane in natural gas, typical values for the deuterium content [9] are about $20 \%$ depleted in D compared to Standard Mean Ocean Water, giving an atom fraction of D of approximately $0.0125 \%$. Since each methane molecule has four hydrogen atoms that could be substituted with deuterium, the corresponding mole fraction of $\mathrm{CH}_{3} \mathrm{D}$ is 
approximately $0.05 \%$, or $x_{\mathrm{CH}_{3} \mathrm{D}}=0.0005$. The fraction of $\mathrm{D}$ is small enough that the effect of multiply substituted species such as $\mathrm{CH}_{2} \mathrm{D}_{2}$ can be neglected.

For the dipole moment of $\mathrm{CH}_{3} \mathrm{D}$, Hollenstein et al. [10] list their own measured values and several literature results, ranging in debye units from roughly $0.0055 \mathrm{D}$ to $0.0057 \mathrm{D}\left(1 \mathrm{D} \approx 3.335641 \times 10^{-30} \mathrm{C} \mathrm{m}\right)$. We adopt a value of $0.0056 \mathrm{D}$ for this analysis.

Substituting these values in Eq. (4) produces $\left\langle p^{2}\right\rangle=1.6 \times 10^{-8} \mathrm{D}^{2}$. Conversion to SI units and substitution in Eq. (3) yields $A_{p}=1.0 \times 10^{-4} \mathrm{~cm}^{3} \mathrm{~K} \mathrm{~mol}^{-1}$. The abovementioned dielectric measurements were performed at temperatures near $300 \mathrm{~K}$, meaning that the contribution of the dipolar term $\left(A_{p} / T\right)$ in Eq. (2) is roughly $3 \times 10^{-7} \mathrm{~cm}^{3} \mathrm{~mol}^{-1}$. The value of $A_{\varepsilon}$ for methane is approximately $6.5 \mathrm{~cm}^{3} \mathrm{~mol}^{-1}$ [5], meaning that the relative contribution of the dipolar term is on the order of $5 \times 10^{-8}$, three orders of magnitude smaller than the relative uncertainty of $A_{\varepsilon}$.

This is only a semiquantitative estimate, since there is significant uncertainty in the $\mathrm{CH}_{3} \mathrm{D}$ mole fraction. However, even under the most "pessimistic" assumptions, the relative contribution of the dipolar term would be no larger than $1 \times 10^{-7}$, which is more than two orders of magnitude smaller than the relative uncertainty of $A_{\varepsilon}$. It is safe to say that, within the precision of current experiments, the small amount of polarity in methane due to the deuterated species can be neglected.

Other substances can be examined in similar ways. We do not find a measured dipole moment for singly deuterated ethane, but the small dipole moment ( 0.01 D) of $\mathrm{CH}_{3} \mathrm{CD}_{3}$ [11] suggests that the relative dipolar contribution to the dielectric constant of ethane is of similar magnitude to that for methane. For HD, both experiment [12] and theory [13] indicate a dipole moment near $0.0009 \mathrm{D}$, which produces an effect ( $A_{p}$ roughly a factor of 80 smaller than for methane) that is negligible compared to the uncertainty of $A_{\varepsilon}$ for $\mathrm{H}_{2}$ [6]. The dipole moment of ${ }^{16} \mathrm{O}^{12} \mathrm{C}^{18} \mathrm{O}$ is approximately $0.0012 \mathrm{D}$ [14], small enough for its effect on the dielectric constant of $\mathrm{CO}_{2}$ to be negligible $\left(A_{p} \approx 3 \times 10^{-5} \mathrm{~cm}^{3} \mathrm{~K} \mathrm{~mol}^{-1}\right)$ despite the relatively high (approximately $0.2 \%$ ) natural abundance of ${ }^{18} \mathrm{O}$. Diatomic molecules such as ${ }^{16} \mathrm{O}^{18} \mathrm{O}$ and ${ }^{14} \mathrm{~N}^{15} \mathrm{~N}$ would be expected to have dipole moments smaller than that of HD, because the post-Born-Oppenheimer effects that produce the moment are inversely proportional to the nuclear masses.

\section{Conclusions}

We have used literature values of dipole moments and isotopic abundances to analyze the effect of naturally occurring isotopologues on precise measurements of the static dielectric constant. For methane, this effect is more than two orders of magnitude smaller than the uncertainty of the best existing measurements. The effect is even less significant for other common gases. Therefore, the analysis of stateof-the-art dielectric-constant data $[4,5,6]$ for these "nonpolar" gases with the assumption of zero dipole moment is valid.

Improvements continue to be made for gas-phase dielectric measurements in metrology $[1,15,16]$, so it is possible that future experiments will achieve lower uncertainties. If the relative uncertainty is reduced by more than one order of magnitude, the effect of isotopologues could become significant, and the framework developed in this paper could be used to assist in data analysis. This framework would also be useful if precision dielectric-constant measurements were made on a sample that was artificially enriched in minor isotopes.

\section{Acknowledgments}

The author thanks Michael Moldover of NIST for helpful discussions and encouragement to publish this work. 


\section{References}

[1] Gaiser C, Zandt T, Fellmuth B (2015) Dielectric-constant gas thermometry. Metrologia 52:S217-S226. https://doi.org/10.1088/0026-1394/52/5/S217

[2] Gavioso RM, Madonna Ripa D, Benyon R, Gallegos JG, Perez-Sanz F, Corbellini S, Avila S, Benito AM (2014) Measuring Humidity in Methane and Natural Gas with a Microwave Technique. International Journal of Thermophysics 35:748-766. https://doi.org/10.1007/s10765-014-1566-8

[3] Harvey AH, Lemmon EW (2005) Method for Estimating the Dielectric Constant of Natural Gas Mixtures. International Journal of Thermophysics 26:31-46. https://doi.org/10.1007/s10765-005-2351-5

[4] Moldover MR, Buckley TJ (2001) Reference Values of the Dielectric Constant of Natural Gas Components Determined with a Cross Capacitor. International Journal of Thermophysics 22:859-885. https://doi.org/10.1023/A:1010731117103

[5] Schmidt JW, Moldover MR (2003) Dielectric Permittivity of Eight Gases Measured with Cross Capacitors. International Journal of Thermophysics 24:375-403. https://doi.org/10.1023/A:1022963720063

[6] May EF, Moldover MR, Schmidt JW (2009) Reference measurements of hydrogen's dielectric permittivity. Molecular Physics 107:1577-1585. https://doi.org/10.1080/00268970902980052

[7] Rourke, PMC, Gaiser C, Gao B, Madonna Ripa D, Moldover MR, Pitre L, Underwood RJ (2019) Refractive-index gas thermometry. Metrologia 56:032001. https://doi.org/10.1088/1681-7575/ab0dbe

[8] Levine IN (1978) Physical Chemistry (McGraw-Hill, New York).

[9] Schoell M (1980) The hydrogen and carbon isotopic composition of methane from natural gases of various origins. Geochimica et Cosmochimica Acta 44:649-661. https://doi.org/10.1016/0016-7037(80)90155-6

[10] Hollenstein H, Marquardt RR, Quack M, Suhm, MA (1994) Dipole moment function and equilibrium structure of methane in an analytical, anharmonic nine-dimensional potential surface related to experimental rotational constants and transition moments by quantum Monte Carlo calculations. Journal of Chemical Physics 101:3588-3602. https://doi.org/10.1063/1.467544

[11] Hirota E, Matsumura C (1971) Microwave Spectrum of Ethane. Journal of Chemical Physics 55:981-982. https://doi.org/10.1063/1.1676171

[12] Drakopoulos PG, Tabisz GC (1987) Far-infrared rotational spectrum of HD: Line shape, dipole moment, and collisional interference. Physical Review A 36:5556-5565. https://doi.org/10.1103/PhysRevA.36.5556

[13] Arapiraca AFC, Jonsson D, Mohallem JR (2011) Vibrationally averaged post Born-Oppenheimer isotopic dipole moment calculations approaching spectroscopic accuracy. Journal of Chemical Physics 135:244313. https://doi.org/10.1063/1.3671940

[14] Karlovets EV, Perevalov VI (2015) The influence of isotopic substitution on the effective dipole moment parameters of $\mathrm{CO}_{2}$ molecule. Optics and Spectroscopy 119:16-21. https://doi.org/10.1134/S0030400X15070139

[15] Guenz C, Gaiser C, Richter M (2017) Concept of a new technique for the combined determination of dielectric and density virial coefficients. Measurement Science and Technology 28:027002. https://doi.org/10.1088/1361-6501/aa53a4

[16] Gaiser C, Fellmuth B (2018) Polarizability of Helium, Neon, and Argon: New Perspectives for Gas Metrology. Physical Review Letters 120:123203. https://doi.org/10.1103/PhysRevLett.120.123203

About the author: Allan H. Harvey is a chemical engineer in the Applied Chemicals and Materials Division at NIST, where he develops models for thermophysical properties of pure fluids and mixtures, with an emphasis on aqueous systems and systems of importance for metrology. The National Institute of Standards and Technology is an agency of the U.S. Department of Commerce. 